\title{
Product-limit estimators of the gap time distribution of a renewal process under different sampling patterns
}

\author{
Richard D. Gill · Niels Keiding
}

Received: 29 September 2009 / Accepted: 23 February 2010 / Published online: 23 March 2010

C) The Author(s) 2010. This article is published with open access at Springerlink.com

\begin{abstract}
Nonparametric estimation of the gap time distribution in a simple renewal process may be considered a problem in survival analysis under particular sampling frames corresponding to how the renewal process is observed. This note describes several such situations where simple product limit estimators, though inefficient, may still be useful.
\end{abstract}

Keywords Kaplan-Meier estimator · Cox-Vardi estimator · Laslett's line segment problem $\cdot$ Nonparametric maximum likelihood $\cdot$ Markov process

\section{Introduction}

This note is about two classical problems in nonparametric statistical analysis of recurrent event data, both formalised within the framework of a simple, stationary renewal process.

We first consider observation around a fixed time point, i.e., we observe a backward recurrence time $R$ and a forward recurrence time $S$. It is well known that the nonparametric maximum likelihood estimator of the gap-time distribution is the Cox-Vardi estimator (Cox 1969; Vardi 1985) derived from the length-biased distribution of the gap time $R+S$. However, Winter and Földes (1988) proposed to use a product-limit estimator based on $S$, with delayed entry given by $R$. Keiding and Gill (1988) clarified the relation of that estimator to the standard left truncation problem. Unfortunately

\section{R. D. Gill (ه)}

Department of Mathematics, University of Leiden, Leiden, Netherlands e-mail: gill@math.leidenuniv.nl

N. Keiding

Department of Biostatistics, University of Copenhagen, Copenhagen, Denmark 
this discussion was omitted from the published version (Keiding and Gill 1990). Since these simple relationships do not seem to be on record elsewhere, we offer them here.

The second observation scheme considers a stationary renewal process observed in a finite interval where the left endpoint does not necessarily correspond to an event. The full likelihood function is complicated, and we briefly survey possibilities for restricting attention to various partial likelihoods, in the nonparametric case again allowing the use of simple product-limit estimators.

\section{Observation of a stationary renewal process around a fixed point}

Winter and Földes (1988) studied the following estimation problem. Consider $n$ independent renewal processes in equilibrium with underlying distribution function $F$, which we shall assume absolutely continuous with density $f$, minimal support interval $(0, \infty)$, and hazard $\beta(t)=f(t) /(1-F(t)), t>0$. The reason for our unconventional choice $\beta$ for the hazard rate belonging to $F$ will become apparent later. Corresponding to a fixed time, say 0 , the backward and forward recurrence times $R_{i}$ and $S_{i}, i=1, \ldots, n$, are observed; their sums $Q_{i}=R_{i}+S_{i}$ are length-biased observations from $F$, i.e., their density is proportional to $t f(t)$. Let $(R, S, Q)$ denote a generic triple $\left(R_{i}, S_{i}, Q_{i}\right)$. We quote the following distribution results: let $\mu$ be the expectation value corresponding to the distribution $F$,

$$
\mu=\int_{0}^{\infty} u f(u) \mathrm{d} u=\int_{0}^{\infty}(1-F(u)) \mathrm{d} u,
$$

then the joint distribution of $R$ and $S$ has density $f(r+s) / \mu$, the marginal distributions of $R$ and $S$ are equal with density $(1-F(r)) / \mu$, and the marginal distribution of $Q=R+S$ has density $q f(q) / \mu$, the length-biased density corresponding to $f$.

Winter and Földes considered the product-limit estimator

$$
1-\tilde{F}(t)=\prod_{i: Q_{i} \leq t}\left(1-\frac{1}{Y\left(Q_{i}\right)}\right)
$$

where

$$
Y(t)=\sum_{i=1}^{n} I\left\{R_{i}<t \leq R_{i}+S_{i}\right\}
$$

is the number at risk at time $t$. This estimator is the same as the Kaplan-Meier estimator for iid survival data $Q_{1}, \ldots, Q_{n}$ left-truncated at $R_{1}, \ldots, R_{n}$ (Kaplan and Meier 1958; Andersen et al. 1993). Winter and Földes showed that $1-\widetilde{F}$ is strongly consistent for the underlying survival function $1-F$.

We shall show how the derivation of this estimator follows from a simple Markov process model similar to the one used by Keiding and Gill (1990) to study the random truncation model. 
First notice that the conditional distribution of $Q=R+S$ given that $R=r$ has density

$$
\frac{f(q) / \mu}{(1-F(r)) / \mu}, \quad r<q<\infty
$$

that is, intensity (hazard) $f(q) /(1-F(q)), q>r$, which is just the hazard $\beta(q)$ corresponding to the underlying distribution $F$ left-truncated at $r$. Now define corresponding to $(R, S, Q)$ a stochastic process $U$ on $[0, \infty]$ with state space $\{0,1,2\}$ by

$$
U(t)= \begin{cases}0, & t<R, \\ 1, & R \leq t<R+S, \\ 2, & R+S \leq t\end{cases}
$$

Note that it takes in successsion the values 0,1 and 2 . For $U(t)=0$,

$$
\begin{aligned}
P\{U(t+h) & =1 \mid U(u), 0 \leq u \leq t\} \\
& =P\{R \leq t+h \mid R>t\} \\
& =\alpha(h) h+o(h),
\end{aligned}
$$

where $\alpha$ is the hazard rate of the marginal distribution of $R$. For $U(t)=1$ (and hence $R \leq t$ )

$$
\begin{aligned}
P\{U(t+h) & =2 \mid U(u), 0 \leq u \leq t\} \\
& =P\{R+S \leq t+h \mid R=r \leq t, R+S>t\} \\
& =\frac{f(t)}{1-F(t)} h+o(h)
\end{aligned}
$$

by the above result on the conditional hazard of $R+S$ given $R$. For $U(t)=0$,

$$
P\{U(t+h)=2 \mid U(u), 0 \leq u \leq t\}=o(h) .
$$

Other transitions are impossible.

That these conditional probabilities depend on $U(t)$ and $t$ only, but not on $U(u)$, $u<t$, proves that $U$ is a Markov process with intensities

$$
\alpha(t)=\frac{1-F(t)}{\int_{t}^{\infty}(1-F(r)) \mathrm{d} r}
$$

(the marginal hazard of $R$, equal to the residual mean lifetime function of the underlying distribution $F$ ) and

$$
\beta(t)=\frac{f(t)}{1-F(t)},
$$


Fig. 1 Inhomogenous 3-state Markov process, 2 allowed transitions

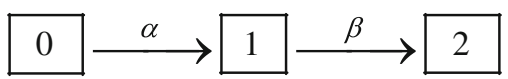

see Fig. 1. The Markov process framework of Keiding and Gill (1990) now indicates that (ignoring information about $F$ in $\alpha$, and just focussing on the transition with rate $\beta$ ) the product limit estimator $1-\widetilde{F}$ is a natural estimator of the survivor function $1-F$ of interest, and consistency and asymptotic normality may be obtained as shown by Keiding and Gill (1990, Sect. 5).

Note that the backwards intensity

$$
\begin{aligned}
\bar{\alpha}(t) & =\alpha(t) \frac{P\{U(t)=0\}}{P\{U(t)=1\}} \\
& =\alpha(t) \frac{P\{R>t\}}{P\{R \leq t<R+S\}} \\
& =\alpha(t) \frac{\mu^{-1} \int_{t}^{\infty}(1-F(r)) \mathrm{d} r}{\mu^{-1} \int_{0}^{t} \int_{t-r}^{\infty} f(r+s) \mathrm{d} s \mathrm{~d} r} \\
& =\frac{1-F(t)}{\int_{t}^{\infty}(1-F(r)) \mathrm{d} r} \frac{\int_{t}^{\infty}(1-F(r)) \mathrm{d} r}{\int_{0}^{t}(1-F(t)) \mathrm{d} r}=\frac{1}{t},
\end{aligned}
$$

the backwards hazard-rate of a uniform distribution on a bounded interval $(0, A)$, $A<\infty$. Since it has been assumed that $R$ has support interval $(0, \infty)$, this shows that the present model may not be interpreted strictly as a left truncation model, which would require that $\bar{\alpha}(t)$ was the backwards hazard rate of some probability distribution on $(0, \infty)$. However, this distinction is not important to our discussion.

The fact that $\bar{\alpha}(t)$ does not depend on $F$ corresponds to Winter and Földes' statement that $(R, S)$ contains no more information than $R+S$ about $F$. This already follows from sufficiency since the joint density of $(R, S)$ is $f(r+s) / \mu$. The likelihood function based on observation of $\left(R_{1}, S_{1}\right), \ldots\left(R_{n}, S_{n}\right)$ is

$$
\mu^{-n} \prod_{i=1}^{n} f\left(r_{i}+s_{i}\right)
$$

from which the NPMLE of $F$ is readily derived as

$$
\widehat{F}(t)=\sum_{i=1}^{n} \frac{I\left\{R_{i}+S_{i} \leq t\right\}}{R_{i}+S_{i}} / \sum_{i=1}^{n} \frac{1}{R_{i}+S_{i}},
$$

that is the Cox-Vardi estimator in the terminology of Winter and Földes (Cox 1969; Vardi 1985).

It follows that the estimator $1-\widetilde{F}$ is not NPMLE. The important difference between the situation here and that of the random truncation model studied by Keiding and Gill (1990, Sect. 3) is that not only the intensity $\beta(t)$, but also $\alpha(t)$ depends only on the estimand $F$. 
As already mentioned, weak convergence of $1-\widetilde{F}$ is immediate from Keiding and Gill (1990, Sect. 5). In particular, in order to achieve the extension to convergence on $[0, M]$ it should be required that

$$
\int_{0}^{\varepsilon} \mathrm{d} \Phi(s) / \nu_{2}(s)<\infty
$$

in the terminology of Keiding and Gill (1990, Sect. 5c), and using $\mathrm{d} \Phi(t)=\beta(t) \mathrm{d} t$ and

$$
v_{2}(t)=P\{U(t)=1\}=\int_{0}^{t} \frac{1-F(s)}{\mu} \frac{1-F(t)}{1-F(s)} \mathrm{d} s=\frac{t}{\mu}(1-F(t)),
$$

the integrability condition translates into

$$
\int_{0}^{\varepsilon} \frac{\beta(t)}{P\{U(t)=1\}} \mathrm{d} t<\infty
$$

or finiteness of $E(1 / X)$ where $X$ has the underlying ("length-unbiased") interarrival time distribution $F$. It may easily be seen from Gill et al. (1988) that the same condition is needed to ensure weak convergence of the Cox-Vardi estimator.

A variation of the observation scheme of this section would be to allow also right censoring of the $S_{i}$. This can be immediately included in the Markov-process/counting process approach leading to the inefficient product-limit type estimator $1-\widetilde{F}$; the delayed-entry observations $S_{i}$ are simultaneously right-censored. See Vardi (1985, 1989) and Asgharian et al. (2002) for treatment of the full non-parametric maximum likelihood estimator of $F$, extending the Cox-Vardi estimator to allow right censoring.

Other ad hoc estimators and the rich relationships with a number of other important non-parametric estimation problems are discussed by Denby and Vardi (1985) and Vardi (1989).

\section{Observation of a stationary renewal process in a finite interval}

We consider again a stationary renewal process on the whole line and assume that we observe it in some interval $\left[t_{1}, t_{2}\right]$ determined independently of the process. Nonparametric estimation of the gap time distribution $F$ was definitively discussed by Vardi (1982) in discrete time and by Soon and Woodroofe (1996) in continuous time. Cook and Lawless (2007, Chap. 4) surveyed the general area of analysis of gap times emphasizing that the assumption of independent gap times is often unrealistic.

We shall here nevertheless work under the assumption of the simplest possible model as indicated above. Because the nonparametric maximum likelihood estimator is computationally involved it may sometimes be useful to calculate less efficient alternatives, and there are indeed such possibilities. 
Under the observation scheme indicated above we may have the following four types of elementary observations

1. Times $x_{i}$ from one renewal to the next, contributing the density $f\left(x_{i}\right)$ to the likelihood.

2. Times from one renewal $T$ to $t_{2}$, which are right-censored versions of 1 , contributing factors of the form $\left(1-F\left(t_{2}-T\right)\right)$ to the likelihood.

3. Times from $t_{1}$ to the first renewal $T$ (forward recurrence times), contributing factors of the form $\left(1-F\left(T-t_{1}\right)\right) / \mu$ to the likelihood.

4. Knowledge that no renewal happened in $\left[t_{1}, t_{2}\right]$, actually a right-censored version of 3., contributing factors of the form $\int_{t_{2}-t_{1}}^{\infty}(1-F(u)) \mathrm{d} u / \mu$ to the likelihood.

McClean and Devine (1995) studied nonparametric maximum likelihood estimation in the conditional distribution given that there is at least one renewal in the interval, i.e., that there are no observations of type 4 .

Our interest is in basing the estimation only on complete or right-censored gap times, i.e., observations of type 1 or 2 . When this is possible, we have simple product-limit estimators in the one-sample situation, and we may use well-established regression models (such as Cox regression) to account for covariates. Peña et al. (2001) assumed that observation started at a renewal (thereby defining away observations of type 3 and 4) and gave a comprehensive discussion of exact and asymptotic properties of product-limit estimators with comparisons to alternatives, building in particular on results of Gill $(1980,1981)$ and Sellke (1988). The crucial point here is that calendar time and time since last renewal both need to be taken into account, so the straightforward martingale approach displayed by Andersen et al. (1993) is not available. Peña et al. also studied robustness to deviations from the assumption of independent gap times.

As noted by Aalen and Husebye (1991) in their attractive non-technical discussion of observation patterns, observation does however often start between renewals. (In the example of Keiding et al. (1998), auto insurance claims were considered in a fixed calendar period). As long as observation starts at a stopping time, inference is still valid, so by starting observation at the first renewal in the interval we can essentially refer back to Peña et al. (2001). A more formal argument could be based on the concept of the Aalen filter, see Andersen et al. (1993, p. 164). The resulting product-limit estimators will not be fully efficient, since the information in the backward recurrence time (types 3 and 4) is ignored. It is important to realize that the validity of this way of reducing the data depends critically on the independence assumptions of the model. Keiding et al. (1998), cf. Keiding (2002) for details, used this fact to base a goodnessof-fit test on a comparison of the full nonparametric maximum likelihood estimator with the product-limit estimator.

Similar terms appear in another model, called the Laslett line segment problem (Laslett 1982). Suppose one has a stationary Poisson process, with intensity $\mu$, of points on the real line. We think of the real line as a calendar time axis, and the points of the Poisson process will be called pseudo renewal times or birth times of some population of individuals. Suppose the individuals have independent and identically distributed lifetimes, each one starting at the corresponding birth time. The corresponding calendar time of the end of each lifetime can of course be called a death time. Now suppose that all we can observe are the intersections of individuals' 
lifetimes (thought of as time segments on the time axis) with an observational window $\left[t_{1}, t_{2}\right]$. In particular, we do not know the current age of an individual who is observed alive at time $t_{1}$. Again we have exactly the same four kinds of observations:

1. Complete proper lifetimes corresponding to births within $\left[t_{1}, t_{2}\right]$ for which death occurred before time $t_{2}$.

2. Censored proper lifetimes corresponding to births within $\left[t_{1}, t_{2}\right]$ for which death occurred after time $t_{2}$.

3. Complete residual lifetimes corresponding to births which occurred at an unknown moment before time $t_{1}$, and for which death occurred after $t_{1}$ and before time $t_{2}$.

4. Censored residual lifetimes corresponding to births which occurred at an unknown moment before time $t_{1}$, for which death occurred after time $t_{2}$, and which are therefore censored at time $t_{2}$.

The number of at least partially observed lifetimes (proper or residual) is random, and Poisson distributed with mean equal to the intensity $\mu$ of the underlying Poisson process of birth times, times the factor

$$
t_{2}-t_{1}+\int_{0}^{\infty}(1-F(y)) \mathrm{d} y .
$$

This provides a fifth, "Poisson", factor in the nonparametric likelihood function for parameters $\mu$ and $F$, based on all the available data. Maximizing over $\mu$ and $F$, the mean of the Poisson distribution is estimated by the observed number of partially observed lifetimes. Thus we find that the profile likelihood for $F$, and the marginal likelihood for $F$ based only on contributions 1.-4., are proportional to one another.

Nonparametric maximum likelihood estimation of $F$ was studied by Wijers (1995) and van der Laan (1996), cf. van der Laan and Gill (1999). Some of their results, and the calculations leading to this likelihood, were surveyed by Gill (1994, p. 190). The nonparametric maximum likelihood estimator is consistent; whether or not it converges in distribution as $\mu$ tends to infinity is unknown, the model has a singularity coming from the vanishing probability density of complete lifetimes just larger than the length of the observation window corresponding to births just before the start of the observation window and deaths just after its end. Van der Laan showed that a mild reduction of the data by grouping or binning leads to a much better behaved nonparametric maximum likelihood estimator. If the amount of binning decreases at an appropriate rate as $\mu$ increases, this leads to an asymptotically efficient estimator of $F$. This procedure can be thought of as regularization, a procedure often needed in nonparametric inverse statistical problems, where maximum likelihood can be too greedy.

Both "unregularized" and regularized estimators are easy to compute with the EM algorithm; and the speed of the algorithm is not so painfully slow as in other inverse problems, since this is still a problem where "root $n$ " rate estimation is possible.

The problem allows, just as we have seen in earlier sections, all the same inefficient but rapidly computable product-limit type estimators based on various marginal likelihoods. Moreover since the direction of time is basically irrelevant to the model, one 
can also look at the process "backwards", leading to another plethora of inefficient but easy estimators. One can even combine in a formal way the censored survival data from a forward and a backward time point of view, which comes down to counting all uncensored observations twice, all singly censored once, and discarding all doubly censored data. (This idea was essentially suggested much earlier by Palmer and Cox, cf. Palmer (1948)). The attractive feature of this estimator is again the ease of computation, the fact that it only discards the doubly censored data, and its symmetry under reversing time. The asymptotic distribution theory of this estimator is of course not standard, but using the nonparametric delta method one can fairly easily give formulas for asymptotic variances and covariances. In practice one could easily and correctly use the nonparametric bootstrap, resampling from the partially observed lifetimes, where again a resampled complete lifetime is entered twice into the estimate.

The Laslett line segment problem has rather important extensions to observation of line segments (e.g., cracks in a rock surface) observed through an observational window in the plane. Under the assumption of a homogenous Poisson line segment process one can write down nonparametric likelihoods, maximize them with the EM algorithm; it seems that regularization may well be necessary to get optimal "root $n$ " behaviour but in principle it is clear how this might be done. Again, we have the same plethora of inefficient but easy product-limit type estimators. van Zwet (2004) studied the behaviour of such estimators when the line segment process is not Poisson, but merely stationary. The idea is to use the Poisson process likelihood as a quasi likelihood, i.e., as a basis for generating estimating equations, which will be unbiased but not efficient, just as in parametric quasi-likelihood. Van Zwet shows that this procedure works fine. Coming full circle, one can apply these ideas to the renewal process we first described in this section, and the other models described in earlier sections. All of them generate stationary line segment processes observed through a finite time window on the line. Thus the nonparametric quasi-likelihood approach can be used there too. Since in the renewal process case we are ignoring the fact that the intensity of the point process of births equals the inverse mean life-time, we do not get full efficiency. So it is disputable whether it is worth using an inefficient ad-hoc estimator which is difficult to compute when we have the options of Soon and Woodroofe's fully efficient (but hard to compute) full nonparametric maximum likelihood estimator, and the many inefficient but easy and robust product-limit type estimators of this paper.

Acknowledgements This research was partially supported by a grant (RO1CA54706-12) from the National Cancer Institute and by the Danish Natural Sciences Council grant 272-06-0442 "Point process modelling and statistical inference".

Open Access This article is distributed under the terms of the Creative Commons Attribution Noncommercial License which permits any noncommercial use, distribution, and reproduction in any medium, provided the original author(s) and source are credited.

\section{References}

Aalen OO, Husebye E (1991) Statistical analysis of repeated events forming renewal processes. Stat Med 10:1227-1240

Andersen PK, Borgan Ø, Gill RD, Keiding N (1993) Statistical models based on counting processes. Springer Verlag, New York 
Asgharian M, Wolfson DB, M'lan CE (2002) Length-biased sampling with right censoring: an unconditional approach. J Am Stat Assoc 97:201-209

Cook RJ, Lawless JF (2007) The statistical analysis of recurrent events. Springer Verlag, New York

Cox DR (1969) Some sampling problems in technology. In: Johnson NL, Smith H Jr New developments in survey sampling. Wiley, New York, pp 506-527

Denby L, Vardi Y (1985) A short-cut method for estimation in renewal processes. Technometrics 27: 361-373

Gill RD (1980) Nonparametric estimation based on censored observations of a Markov renewal process. Zeitschrift für Wahrscheinlichkeitstheorie und verwandte Gebiete 53:97-116

Gill RD (1981) Testing with replacement and the product-limit estimator. Ann Stat 9:853-860

Gill RD (1994) Lectures on survival analysis. Lecture notes in Mathematics, vol 1581. Springer, New York, pp 115-241

Gill RD, Vardi Y, Wellner JA (1988) Large sample theory of empirical distributions in biased sampling models. Ann Stat 16:1069-1112

Kaplan EL, Meier P (1958) Non-parametric estimation from incomplete observations. J Am Stat Assoc 53:457-481

Keiding N (2002) Two nonstandard examples of the classical stratification approach to graphically assessing proportionality of hazards. In: Huber-Carol C, Balakrishnan N, Nikulin MS, Mesbah M Goodnessof-fit tests and model validity. Birkhäuser, Boston, pp 301-308

Keiding N, Andersen C, Fledelius P (1998) The Cox regression model for claims data in non-life insurance. ASTIN Bull 28:95-118

Keiding N, Gill RD (1988) Random truncation models and Markov processes. Report MS- R8817. Centre for Mathematics and Computer Science, Amsterdam

Keiding N, Gill RD (1990) Random truncation models and Markov processes. Ann Stat 18:582-602

Laslett GM (1982) The survival curve under monotone density constraints with application to two-dimensional line segment processes. Biometrika 69:153-160

McClean S, Devine C (1995) A nonparametric maximum likelihood estimator for incomplete renewal data. Biometrika 82:791-803

Palmer RC (1948) The dye sampling method of measuring fibre length distribution (with an appendix by R.C. Palmer and D.R. Cox). J Text Inst 39:T8-T22

Peña EA, Strawderman RL, Hollander M (2001) Nonparametric estimation with recurrent event data. J Am Stat Assoc 96:1299-1315

Sellke T (1988) Weak convergence of the Aalen estimator for a censored renewal process. In: Gupta S, Berger J Statistical decision theory and related topics IV, vol 2. Springer-Verlag, New York, pp 183-194

Soon G, Woodroofe M (1996) Nonparametric estimation and consistency for renewal processes. J Stat Plan Inference 53:171-195

van der Laan MJ (1996) Efficiency of the NPMLE in the line-segment problem. Scand J Stat 23:527-550

van der Laan MJ, Gill RD (1999) Efficiency of NPMLE in nonparametric missing data models. Math Methods Stat 8:251-276

van Zwet EW (2004) Laslett's line segment problem. Bernoulli 10:377-396

Vardi Y (1982) Nonparametric estimation in renewal processes. Ann Stat 10:772-785

Vardi Y (1985) Empirical distributions in selection bias models. Ann Stat 13:178-203

Vardi Y (1989) Multiplicative censoring, renewal processes, deconvolution and decreasing density: nonparametric estimation. Biometrika 76:751-761

Wijers BJ (1995) Consistent nonparametric estimation for a one-dimensional line-segment process observed in an interval. Scand J Stat 22:335-360

Winter BB, Földes A (1988) A product-limit estimator for use with length-biased data. Can J Stat 16: 337-355 